\title{
Cambios antropométricos en estudiantes del curso avanzado de combate en la Escuela Militar de Cadetes
}

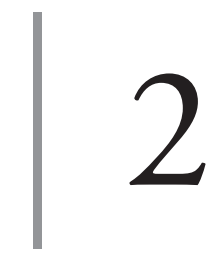

https://doi.org/10.21830/9789585241466.02

Daniel Fernando Aparicio Gómez ${ }^{1}$

\section{Resumen:}

Objetivo: el objetivo del presente estudio fue determinar los cambios antropométricos del personal militar que participa de un Curso Avanzado de Combate y sus repercusiones significativas en su constitución física como indicador de la mejoría o no de la aptitud física del individuo. Materiales y métodos: se realizó un estudio longitudinal con 69 militares (56 hombres y 13 mujeres) en formación, pertenecientes a la Escuela Militar de Cadetes "General José María Córdova”, del Ejército de Colombia. Una vez diligenciados los consentimientos informados, se realizó una evaluación antropométrica previa a la asistencia del CAC y una posterior, que incluyó medición de talla, peso, circunferencia de cintura. Asimismo, se evaluó la composición corporal por medio de bioimpedancia eléctrica (Seca 525) y se estableció mediante el uso del programa estadístico SPSS Ver. 21 el comportamiento de la normalidad de los datos. Posteriormente se estableció la significancia estadística de los datos usando el método de T de Student para datos pareados. Resultados: las variables de peso, Índice de Masa Corporal (IMC), masa grasa relativa (MGR), masa grasa absoluta (MGA), tejido adiposo visceral (TAV), agua extracelular (AEC) y ángulo de fase (AF) tuvieron cambios estadísticamente significativos, es decir que la carga física que impone el CAC repercute en los cambios de las variables mencionadas. Por el contrario, no se observan modificaciones estadísticamente significativas respecto a la masa libre de grasa (MLG), la masa de músculo esquelético (ME), el agua corporal total (AT) ni el perímetro de cintura (PC). Conclusiones: si bien la carga física que implica un CAC de ocho semanas produce cambios en todas las

1 Msc. en Ciencias y Tecnologías del Deporte y la Actividad Física. Médico especialista en Medicina del Deporte. Médico especialista en Gerencia de la Salud Ocupacional. Grupo de Investigaciones Renfimil, Escuela Militar de Cadetes “General José María Córdova”, Bogotá, Colombia. Contacto: daniel.aparicio@idrd.gov.co - https://orcid.org/0000-0001-5574-7580 
variables antropométricas evaluadas por bioimpedancia eléctrica, solo algunas de ellas presentan cambios estadísticamente significativos, pero no se prueban respecto a las variables masa libre de grasa, músculo esquelético, agua total ni perímetro de cintura.

Palabras clave: porcentaje de grasa corporal, índice de masa grasa, indicadores de adiposidad, bioimpedancia eléctrica, antropometría, composición corporal, Índice de Masa Corporal.

\section{Introducción}

Los alumnos de la Escuela Militar de Cadetes "General José María Córdova" (Esmic) deben enfrentar como parte de su formación el Curso Avanzado de Combate (CAC). Se trata de un programa de instrucción y entrenamiento en el que los estudiantes de las escuelas de formación del Ejército Nacional se preparan y certifican como comandantes de unidades tácticas y terreno, para liderar escuadras y pequeñas unidades en el área técnica, táctica, humanística y física, en ambientes simulados de combate (1, 2). El CAC permite que el alumno desarrolle las competencias y habilidades necesarias para preservar su vida y la de sus superiores y subalternos, al tiempo que alcance el objetivo de la misión en una situación operativa lo más cercano a los escenarios reales. El curso tiene en cuenta dos aspectos, uno académico y otro físico, y de los resultados que alcance el cadete dependerá si puede o no ascender de rango militar, lo cual impone una cuota de gran desafío.

En lo que respecta al área de desempeño físico, durante ocho semanas el estudiante se enfrenta a situaciones que ponen a prueba las condiciones fisiológicas y físicas que ha desarrollado en los seis niveles previos al curso, tales como la fuerza, la resistencia y la velocidad, además de capacidades condicionales y la flexibilidad, el equilibrio, la agilidad y la movilidad, así como capacidades coordinativas (3). Estas capacidades, además de que no son las mismas en todos los alumnos, varían por la alta exigencia del curso, el poco tiempo para lograrlo, el ambiente climático hostil en el que se realiza, el atuendo empleado y el peso adicional que representa el equipo de campańa.

En este punto es importante mencionar que, aunque el ejercicio como tal, en poblaciones no militares, provee beneficios para la salud (4), en situa- 
ciones operacionales y climáticas adversas puede generar impactos indeseados en las personas que reciben el entrenamiento, como argumenta la revisión sistemática de Gómez et al. (5) sobre varios entes militares que enfrentan cursos cortos de entrenamiento. Asimismo, se ha documentado que el ejercicio puede generar cambios en la composición corporal de los sujetos (6, 7) y en otros aspectos de la aptitud física, que no son tema de este capítulo.

Los resultados de este trabajo evidencian los efectos del CAC sobre las condiciones antropométricas de los alumnos y, por lo tanto, permiten evaluar de forma indirecta los componentes del curso. Este análisis es necesario para que a mediano o largo plazo se realicen las adecuaciones que sean necesarias al curso con el fin de que los objetivos de formación se logren sin detrimento del bienestar de los participantes.

En Colombia, al igual que en muchos otros países, tradicionalmente se han utilizado criterios antropométricos, como los índices basados en el peso, la talla y su relación, es decir, el Índice de Masa Corporal (IMC), para definir la obesidad, la desnutrición y los trastornos alimentarios. Su cálculo se realiza dividiendo el peso de una persona en kilos por el cuadrado de su talla en metros $\left(\mathrm{kg} / \mathrm{m}^{2}\right)$.

Sin embargo, estas variables tienen poca sensibilidad para monitorear la respuesta que tiene el paciente, deportista o, en este caso, el alumno de la escuela de formación al entrenamiento que recibe, de manera que es ideal evaluar la composición corporal segmentaria porque permite cualificar este proceso. Adicionalmente, esta disposición de los diferentes compartimentos, como la grasa corporal y su distribución en el cuerpo, necesita una mayor atención en su relación con la etiología de las enfermedades cardiovasculares, la hipertensión y la diabetes tipo 2, enfermedades crónicas que tienen su "periodo de incubación” durante la niñez y la adolescencia, según la literatura más reciente (8).

El IMC proporciona la medida más rápida para determinar el sobrepeso y la obesidad en una población, y se usa de forma indiscriminada para ambos géneros y para los adultos de todas las edades. No obstante, desde el punto de vista de la medicina del deporte, la nutrición, entre otras áreas 
del conocimiento, es una medida demasiado sucinta y en algunos casos aparente e inadecuada, que no permite determinar un estado nutricional del sujeto evaluado. Diversos estudios evidencian que el IMC muestra una baja confiabilidad para estimar adiposidad a nivel individual, particularmente en hombres y cuando el IMC es menor de $30 \mathrm{~kg} / \mathrm{m}^{2}$ (9).

Esta es la razón por la cual el Centro de Investigación de la Cultura Física (Cicfi) de la Esmic del Ejército Nacional de Colombia se dio a la tarea de determinar los diferentes componentes de la composición corporal de un personal de cadetes militares, el efecto de su entrenamiento en el CAC y el impacto que tiene sobre su estructura. Con este propósito se usó la bioimpedanciometría eléctrica aplicando de forma exhaustiva los protocolos de su uso y su incidencia sobre los diferentes compartimentos del sujeto, antes y al final del curso.

\section{Sensibilidad y especificidad de la impedancia bioeléctrica en la antropometría}

El estudio de la composición corporal es un tema de interés creciente que puede ser utilizado tanto para la investigación como para fines clínicos. Para los profesionales de las ciencias de la salud es importante conocer las características de los diferentes métodos de evaluación y análisis de la composición corporal, ya que cambios en esta guardan relación directa con la salud y el riesgo de enfermedades. Actualmente, los métodos de análisis de la composición corporal son divididos en tres grupos: el directo, los indirectos y los doblemente indirectos. El método directo es la disección de cadáveres. Entre los indirectos encontramos la tomografía axial computarizada (TAC), la resonancia magnética nuclear (RMN), la absorciometría dual de rayos $\mathrm{X}$ (DEXA) y la pletismografía; y en referencia a los métodos doblemente indirectos destacan la antropometría y la impedancia bioeléctrica (BIA, por su sigla en inglés) (10).

Históricamente, el estudio de la composición corporal se ha establecido más por lo que se ha logrado realizar, que por lo que se ha querido investigar, 
siendo la limitante fundamental las diferentes técnicas desarrolladas (11). El método ideal para estudiar la composición corporal de un individuo debería analizar por separado todos y cada uno de los compartimentos que integran el organismo humano (masa grasa, masa ósea, masa muscular, agua, etc.); es por eso que el método más completo hasta la fecha es el análisis del cadáver. Estos estudios se realizaron entre 1945 y 1956 en los cadáveres de cinco hombres y una mujer, y aunque la diferencia entre ellos en cuanto al tejido graso era considerable, todos mostraron en relación con los tejidos libres de grasa unos valores relativamente constantes de $73 \%$ de agua, alrededor de $20 \%$ de proteína y cerca de $69 \mathrm{mmol} \mathrm{K} / \mathrm{kg}$ (12). Hasta el momento, ninguno de los métodos para evaluar la composición corporal se puede hacer de manera directa en el sujeto vivo, por lo cual es necesario derivarla midiendo por separado las propiedades corporales, de ahí que en la práctica todas las técnicas presenten dos tipos de error: 1) metodológico, cuando se lleva a cabo la recolección del dato primario, y 2) en los supuestos que se asumen cuando el dato primario es convertido en el resultado final. Además, la magnitud relativa de estos errores varía entre las técnicas (13).

La impedancia bioeléctrica se presenta entonces como un método sencillo, económico, fácil de utilizar y que proporciona una mayor información en la práctica para el seguimiento y evaluación de los pacientes. Es una de las técnicas no invasivas y portátiles que se han utilizado desde hace más de una década para el análisis de la composición corporal; sin embargo, la impedancia bioeléctrica no mide la composición corporal directamente, sino que la obtiene con base en dos parámetros: la resistencia corporal y la reactancia $(14,15)$.

El análisis por BIA mide la resistencia o impedancia del cuerpo a una corriente eléctrica pequeña, indetectable para el sujeto. La BIA se fundamenta en el hecho de que el tejido magro contiene un alto nivel de agua y electrolitos, y por lo tanto actúa como un conductor eléctrico y la grasa como aislante (13), asumiendo que el agua corporal total es una proporción fija de la masa libre de grasa (73\%) (16). La bioimpedancia eléctrica se fundamenta en la oposición de las células, los tejidos o líquidos corporales 
al paso de una corriente eléctrica generada por el aparato. La masa libre de grasa, como los músculos, huesos, etc., tiene la mayor parte de fluidos y electrolitos corporales.

Una vez que se ha obtenido el valor de la masa libre de grasa, la masa grasa se calcula a partir de la diferencia con el peso corporal total (15). De acuerdo con la declaración de la Conferencia de los Institutos Nacionales de Evaluación en Tecnología de Salud (Bethesda, Maryland, 12-14 de diciembre de 1994), en relación con la utilización del BIA en el estudio de la composición corporal, se determinó que esta técnica es más precisa que el IMC y quizá más precisa que la medición de los pliegues cutáneos para la estimación comparativa de la masa grasa (17).

De manera que este aparato proporciona una estimación directa del agua corporal total y, con base en esta información, se calcula de forma indirecta la masa libre de grasa y la masa grasa a través de fórmulas preestablecidas. La fiabilidad y precisión de este método puede variar por distintos factores, como el tipo de instrumento, puntos de colocación de los electrodos, nivel de hidratación, alimentación, ciclo menstrual, temperatura del ambiente y la ecuación de predicción utilizada; sin embargo, en general es cercana a $r^{2}=0,84$ en comparación con la DEXA (18). Por estas razones, se deben observar algunos cuidados antes de realizar la impedancia bioeléctrica, para evitar la producción de errores, como no comer o beber cuatro horas antes de la prueba, no hacer ejercicios doce horas antes, orinar treinta minutos antes, no ingerir alcohol veinticuatro horas antes y no haber usado diuréticos en los últimos siete días (19).

La variable impedancia $(Z)$, medida en ohmios, es la raíz cuadrada de la suma de los cuadrados de la resistencia $(\mathrm{R})$ y la reactancia $\left(\mathrm{Xc}_{\mathrm{c}}\right)$, y es dependiente de la frecuencia. La $\mathrm{R}$ es la oposición pura de un conductor biológico al flujo de una corriente eléctrica alterna, mientras que la Xc es el efecto de la resistencia debido a la capacitancia — almacenamiento de carga eléctrica en un condensador-, producida por las interfaces de los tejidos y las membranas celulares. La capacitancia hace que la corriente deje atrás la tensión, de manera que se crea un cambio de fase. Este cambio se cuantifica 
geométricamente como la transformación angular de la relación de la Xc a la $\mathrm{R}$, o el ángulo de fase (20).

Por su parte, el ángulo de fase (AF) es el parámetro de la BIA que se ha establecido para diagnosticar la desnutrición y hacer el pronóstico clínico, ambos asociados con cambios en la integridad de la membrana celular y las alteraciones en el balance de líquido. El AF expresa cambios en la cantidad y la calidad de la masa de los tejidos blandos (es decir, permeabilidad de la membrana celular e hidratación). Una gran cantidad de ensayos clínicos proponen el AF como un marcador pronóstico útil en condiciones clínicas, como en cirrosis hepática, en cáncer de mama, colon, páncreas, pulmón; también se observó en pacientes con VIH positivos y quirúrgicos con una asociación positiva entre el AF y la supervivencia (21). Aún no se ha llegado a un consenso de sus valores normales, pero el comportamiento general del AF tiene correlación negativa con la edad, y hubo correlación positiva entre el IMC y el ángulo de fase en los sujetos con bajo peso y de peso normal.

Entre otros, algunos ejemplos de investigaciones que utilizaron este método de evaluación de la composición corporal son los estudios realizados en 2014 por Madsen et al. (22), en hombres jóvenes saludables de India, para verificar los efectos de un programa de ciclismo sobre la composición corporal de estos sujetos. Por Saladino (23) en pacientes con disturbios alimentarios, para evaluar los cambios en la composición corporal de estos durante el tratamiento. En 2015 por Camina-Martin et al. (24), en hombres mayores con y sin demencia, para comparar la antropometría y la impedancia bioeléctrica, así como para verificar la relación entre la demencia y la composición corporal. Finalmente, en el mismo año por Esco et al. (25), en atletas universitarias del sexo femenino, para evaluar la fiabilidad de este método para medir la composición corporal en los distintos segmentos corporales.

Por su parte, en 1994 Núñez et al., estudiando la composición corporal en mujeres jóvenes, reportaron la buena correlación entre el BIA y la antropometría, por lo cual propusieron esta técnica como una alternativa para medir la composición corporal en poblaciones homogéneas con un peso estable 
(26). En contraste, otros autores no recomiendan el uso de esta técnica en personas obesas o muy delgadas (27). La información disponible indica que la impedancia bioeléctrica no es útil para medir los cambios agudos en la grasa corporal de los individuos, aunque sí puede caracterizar los cambios a largo plazo (17), razón por la cual algunos investigadores plantean actualmente que el valor fundamental de la BIA consiste en la vigilancia epidemiológica para estimar la masa magra (13).

Las principales ventajas de este método son que permite diferenciar la grasa y el tejido magro, monitorea la composición de la pérdida de peso; algunos modelos proporcionan un análisis segmentario, simple y fácil de ejecutar, altamente confiable para hacer estudios a gran escala; permite imprimir los resultados inmediatamente, la mayoría son equipos portátiles, no invasivos, con riesgo muy bajo, de bajo costo — comparados con otros métodos de alta tecnología-, y con un valor predictivo elevado (extensas validaciones), además presenta excelente consistencia para mediciones repetidas (27). No obstante, también presenta desventajas: no se recomienda su uso en pacientes con marcapaso; no es tan preciso como los modelos gold standard de cuatro compartimentos; no hay versiones disponibles para niños menores de 5 años; no se recomienda para pacientes con trastornos en el equilibrio hidroelectrolítico y en su mayoría los pacientes deben estar en condiciones de colocarse de pie sobre la plataforma en los modelos pie; tiene limitaciones de aplicación en pacientes que presentan retención de líquidos, edemas periféricos, problemas hidrostáticos o que hagan uso de medicación diurética, o con algún tipo de amputación o déficit anatómico de alguna extremidad; en deportistas no es un método adecuado porque en esta población tiene un error del $3 \%$, lo cual es demasiado elevado como para reportar al deportista su estado de salud. Además de esto, un ligero cambio en el lugar de los electrodos puede producir una variabilidad del $2 \%$ de los resultados en diferentes días $(27,16)$. 


\section{Metodología}

Estudio de tipo longitudinal, con cadetes de séptimo nivel de la Esmic que para el segundo semestre de 2018 se encontraban seleccionados para participar en el Curso Avanzado de Combate como requisito de graduación para su año lectivo 2018. Con un universo de 120 cadetes, se hizo una selección aleatoria simple de 80 sujetos, de acuerdo con las instrucciones que se dieron al comandante de grupo de los cadetes. Al final se contó con un total de 69 sujetos sanos (13 mujeres y 56 hombres) mayores de 18 años y con una edad máxima de 24 años (57,5\% de la población total del curso), de manera que se garantizó una muestra estadísticamente representativa. Los criterios de inclusión aplicados fueron: alumnos en formación militar de séptimo nivel; hombres y mujeres sanos con edades entre 18 y 24 años que hubieran cursado y aprobado los niveles anteriores obligatorios de la Esmic. Los criterios de exclusión fueron: cadetes que no firmaron consentimiento informado; que en el transcurso del CAC fueron retirados o no completaron el curso de forma satisfactoria; los cadetes que no tuvieron las dos evaluaciones antropométricas pre y post curso o los sujetos que en el procesamiento, selección y análisis de datos presentaron valores incompletos o con fallas en la digitación. Asimismo, se aplicó el protocolo de realización de BIA, de forma exhaustiva, con las recomendaciones mencionadas en la literatura, y con el fin de evitar la mayor cantidad de sesgos, se hizo en ayunas, a primera hora del día (entre 4:30 y 6:00 de la mañana), en ambas tomas pre y post curso.

Para este estudio se realizaron las siguientes fases:

1. Identificación del personal que asistiría al CAC en el segundo semestre de 2018.

2. Sensibilización de la población, explicándole por qué y para qué realizar el estudio.

3. De manera individual se informó a los cadetes sobre los riesgos y beneficios para participar voluntariamente en el estudio.

4. Los sujetos firmaron el consentimiento informado y se incluyeron voluntariamente en el estudio. 
5. Realización de antropometría por BIA antes de la salida al CAC de acuerdo con los criterios de inclusión y exclusión, además del protocolo de aplicación.

6. Realización de antropometría por BIA posterior al CAC entre los primeros 2 y 5 días posteriores a la finalización del curso. Nuevamente, también en este proceso se tuvo en cuenta el protocolo de aplicación.

En total se evaluaron once variables (dependientes) de forma pre y post Curso Avanzado de Combate (tabla 1).

Tabla 1. Variables y abreviaturas

\begin{tabular}{lc}
\hline \multicolumn{1}{c}{ Variables } & Abreviatura \\
\hline Peso & Peso \\
Índice de Masa Corporal & IMC \\
Masa de Grasa Relativa & MGR \\
Masa de Grasa Absoluta & MGA \\
Tejido Adiposo Visceral & TAV \\
Masa Libre de Grasa & MLG \\
Masa de Músculo Esquelético & ME \\
Agua Corporal Total & AT \\
Agua Extracelular & AEC \\
Perímetro de Cintura & PC \\
Ángulo de fase & AF \\
\hline
\end{tabular}

Fuente: Original del autor.

\section{Resultados}

Se evaluó un total de 69 sujetos pertenecientes a la Esmic. La distribución por género fue de 13 mujeres (18,5\%) y 56 hombres (81,5\%). El promedio de edad de la población en general fue de 21,15 $\pm 1,14$ años, con una talla promedio de $1,68 \pm 0.67$ centímetros (mínima: 1,51 y máxima 
$1,81 \mathrm{~cm})$. Las once variables antropométricas analizadas se encuentran con sus respectivos datos descriptivos en la tabla 2 .

Tabla 2. Descriptivos de variables antropométricas del personal de cadetes de la Esmic. Valores pre (1) y post (2) Curso Avanzado de Combate ( $\mathrm{n}=69)$

\begin{tabular}{cccc}
\hline Variable & Media & $\begin{array}{c}\text { Desviación } \\
\text { típica }\end{array}$ & $\begin{array}{c}\text { Error típico } \\
\text { de la media }\end{array}$ \\
\hline Peso 1 & 66,574 & 8,8313 & 1,0632 \\
Peso 2 & 64,323 & 7,7093 & 0,9281 \\
\hline IMC 1 & 23,3165 & 2,16537 & 0,26068 \\
IMC 2 & 22,5168 & 1,80848 & 0,21772 \\
\hline MGR 1 & 17,9713 & 7,47749 & 0,90018 \\
MGR 2 & 15,3980 & 6,37592 & 0,76757 \\
\hline MGA 1 & 12,0994 & 5,40873 & 0,65113 \\
MGA 2 & 9,9172 & 4,13968 & 0,49836 \\
\hline TAV 1 & 1,4096 & 0,42321 & 0,05095 \\
TAV 2 & 0,9961 & 0,25037 & 0,03014 \\
\hline MLG 1 & 54,4745 & 7,66051 & 0,92222 \\
MLG 2 & 54,4059 & 7,42892 & 0,89434 \\
\hline ME 1 & 26,3086 & 4,58379 & 0,55182 \\
ME 2 & 25,7114 & 4,09703 & 0,49322 \\
\hline AT 1 & 39,645 & 5,5256 & 0,6652 \\
AT 2 & 39,646 & 5,3657 & 0,6460 \\
\hline AEC 1 & 15,749 & 2,0971 & 0,2525 \\
AEC 2 & 16,070 & 2,1561 & 0,2596 \\
\hline PC 1 & 0,7414 & 0,04806 & 0,00579 \\
PC 2 & 0,7410 & 0,04766 & 0,00574 \\
\hline AF 1 & 7,561 & 0,6411 & 0,0772 \\
AF 2 & 7,226 & 0,6646 & 0,0800 \\
\hline & & &
\end{tabular}

Fuente: Original del autor.

La tabla 2 muestra una diferencia de peso entre la toma pre CAC de $66,57 \pm 8,8 \mathrm{~kg}$ y una post $\mathrm{CAC}$ de $64,32 \pm 7,7 \mathrm{~kg}$, con una diferencia de 2,25 
$\mathrm{kg}$ en promedio menos posterior al curso. De igual forma, un valor de IMC pre CAC de 23,32 $\pm 0,26$ y un IMC post CAC de $22.5 \pm 0.21$, diferencia de 0,79 menor al final del curso.

De manera general se puede observar que se encuentra una disminución de todas las variables, con excepción de los valores de agua total y agua extracelular.

El primer paso fue determinar la normalidad de los datos aplicando las pruebas de Kolmogorov-Smirnov y Shapiro-Wilk, con un intervalo de confianza del $95 \%$. Una vez aplicadas las pruebas se confirmó que todas las variables analizadas en su estadístico son mayores a 0,05 , por lo tanto, su distribución es normal.

De acuerdo con lo anterior se decidió utilizar el Test de la t de Student para una muestra relacionada, con un intervalo de confianza del $95 \%$, es decir un error del $5 \%$, en donde se parte de una Hipótesis nula Ho, en donde se determina que no hay diferencia significativa en los valores, y una hipótesis alternativa $\mathrm{H} 1$, en donde pensamos que sí existe una diferencia significativa.

La interpretación permitió determinar el valor de p, y se rechazó la hipótesis nula Ho.

Se determinó que la Hipótesis alterna H1, es verdadera. Se puede afirmar que las variables de Peso, IMC, MGR, MGA, TAV, AEC y AF tuvieron cambios estadísticamente significativos, es decir la carga física que impone el CAC repercute en los cambios de las variables mencionadas. Por el contrario, no se observan cambios estadísticamente significativos en lo relacionado a la MLG, ME, AT y PC.

\section{Discusión}

Los valores que han logrado una disminución en forma significativa post Curso Avanzado de Combate han sido el peso, el índice de masa corporal, la masa grasa relativa y absoluta, el tejido adiposo visceral, el agua extracelular y el ángulo de fase. En general, todos estos positivos desde el punto de vista 
médico, nutricional y de salud, de manera que contribuyen a disminuir el riesgo cardiovascular, y son ideales de acuerdo con los parámetros de antropometría.

Las variables en las cuales se observa un mayor cambio porcentual se refieren a la masa grasa, tanto en porcentajes como en peso total dentro del organismo. Este cambio se debe fundamentalmente al entrenamiento aeróbico que realizan los alumnos (28), el cual tiene como fuente principal de energía los lípidos, lo cual explica que las variables de peso corporal y de IMC hayan sufrido una disminución significativa, ya que se ha perdido peso por la reducción de la cantidad total de grasa corporal, pero al mismo tiempo la cantidad de agua extracelular, sin encontrar aumento de la masa muscular ni de la masa libre de grasa.

Por su parte, las variables que no presentaron cambios — la masa libre de grasa, el volumen del músculo esquelético, el agua total y el perímetro de cintura - reflejan las condiciones y objetivos más significativos del curso: la preparación técnica, táctica, psicológica y, en un menor grado, física del soldado para las operaciones militares en zonas de combate — generalmente hostiles—, con las particularidades de clima, alimentación, hidratación y condiciones psicológicas adversas. Estas son las condiciones reales que enfrenta el soldado colombiano en sus teatros de operaciones cuando ejerce su carrera luego de la graduación, de allí que el CAC busque básicamente mejorar sus condiciones de resistencia y tolerancia a este tipo de noxas y que desde el punto de vista físico su objetivo no sea lograr la ganancia de masa muscular o potencia de ningún tipo. Se debe recordar que el incremento de la masa muscular está determinado en gran medida por un predominio de la síntesis proteica sobre su destrucción (28), predominio que en el caso de deportistas que entrenan fuerza como una cualidad específica puede llegar a aumentar hasta el 3,9\% la cantidad de proteínas medidas en el posentrenamiento (29). Sin embargo, debido al tipo de entrenamiento que caracteriza el CAC y posiblemente al gran anabolismo proteico que conllevan los ejercicios de resistencia que realizan los alumnos, asociados a altas temperaturas y aportes nutricionales bajos, el aumento de la síntesis proteica puede ser supe- 
rado por los procesos catabólicos que generan la acumulación de sesiones de trabajo continuo aeróbico a lo largo del curso. Como se ve, esto podría explicar que no se vean cambios en estas variables.

En relación con el ángulo de fase, se considera que una exposición aguda (ocho semanas) en sujetos sanos, sin condiciones patológicas previas no impacta los valores de forma significativa, y de igual forma no se vieron reflejados en los efectos finales.

Los resultados de este estudio indican que el contenido estructurado de entrenamiento, elaborado objetivamente y con aplicación de forma aguda, a pesar de las inclemencias del clima, la carga emocional y el estrés de las áreas de operaciones simuladas, permiten a los alumnos de este tipo de cursos presentar variaciones corporales y mejorías en la antropometría, secundarias al tipo de cargas impuestas y asociadas a los modelos nutricionales que acompañan estos procesos. Asimismo, el estudio determinó que las variaciones corporales fueron adecuadas y que de igual forma son parte fundamental de la formación y la realidad del militar en las áreas de operaciones.

Estos resultados serán muy provechosos en un futuro a corto, mediano y largo plazo, siempre y cuando se pueda tecnificar de manera más avanzada y objetiva la aplicación de las cargas en este tipo de cursos, orientados cada vez más a mejorar las condiciones antropométricas de nuestros soldados.

Finalmente, en relación con la diferenciación entre hombres y mujeres cabe señalar que es importante tener en cuenta esta variable para futuros estudios y aumentar el número de sujetos de género femenino, ya que en este caso solo participaron trece mujeres y las conclusiones con esta muestra no se podrían generalizar en la población militar femenina.

\section{Conclusiones}

Se puede concluir que después de ocho semanas de entrenamiento, las variables de peso, Índice de Masa Corporal, masa grasa tanto relativa como absoluta, tejido adiposo visceral, agua extracelular y ángulo de fase presentaron cambios estadísticamente significativos. Es decir, se encontró 
que la carga física que impone el CAC repercute en los cambios de las variables mencionadas. De igual forma, no se hallaron cambios estadísticamente significativos respecto a la masa libre de grasa, músculo esquelético, agua total y perímetro de cintura. Además de los parámetros antropométricos descritos, es necesario que en futuros estudios se analicen los aportes nutricionales e hídricos con el fin de fortalecer estas variables, si fuera necesario, así como diferenciar los impactos por género.

\section{Agradecimientos}

Esta investigación ha sido soportada con recursos de la convocatoria interna en investigación del Comando de Educación y Doctrina del Ejército de Colombia, dentro del proyecto titulado "Cambios en las variables fisiológicas y biomecánicas debido al curso Avanzado de Combate (CAC)”. Agradezco a las directivas y personal integrante de la Escuela Militar de Cadetes "General José María Córdova" por el apoyo en el desarrollo del proyecto de investigación. No existe ningún conflicto de interés por parte del investigador.

\section{Referencias}

1. Ejército Nacional de Colombia. En desarrollo Curso Avanzado de Combate [Internet]; 2016 [citado 2018 febrero 21]. Disponible en: https://www.ejercito.mil.co/?idcategoria $=393662$

2. Ejército Nacional de Colombia. Curso Avanzado de Combate en la Escuela de Suboficiales del Ejército Nacional de Colombia [video en internet]; 2015 [citado 2018 febrero 21]. Disponible en: https://www.youtube.com/watch?v=LuDcH0jZ1Kw.

3. Zuluaga J, Rincón Tabares AS, Roa Quintero MA. Trabajo de grado como requisito para el título de educador físico militar [tesis]. Bogotá, D. C.: Escuela Militar de Cadetes "General José María Córdova”; 2017.

4. Jacoby E, Bull F, Neiman A. Cambios acelerados del estilo de vida obligan a fomentar la actividad física como prioridad en la Región de las Américas. Revista Panamericana de Salud Pública. 2007; 14 (4): 223-225.

5. Aparicio Gómez D F, Castro Jiménez LE, García-Muñoz AI, Cubides Amézquita JR, Puentes Salazar, AM. Revisión sistemática de la repercusión fisiológica de los cursos 
militares operacionales cortos para el soldado. Rev Cient Gen José María Córdova. 2019 abril-junio; 17 (26), 2019, 433-451.

6. Brandão de Albuquerque Filho NJ, Mendes Rebouças G, Araújo Ferreira Matos V, de Mello Salgueiro CC, Knackfuss MI, de Medeiros HJ. Efecto del entrenamiento concuM rrente en la composición corporal y perfil lipídico en adolescentes con sobrepeso. Revista de Educación Física. 2018; 46 (1). Disponible en https://revistadeeducacionfisica.com/ articulo/efecto-del-entrenamiento-concurrente-en-la-composicion-corporal-y-perfil-lipidico-en-adolescentes-con-sobrepeso-2199-sa-258615b143d645

7. Cortés Fernández, Camargo IY, Botero Rosas, D. Caracterización de la composición corporal del personal militar después de realizar el Curso Avanzado de Combate en el Ejército Nacional de Colombia. Rev Cient Gen José María Córdova. 2018 enero-junio; 16(22), 93-106. DOI: http://dx.doi.org/10.21830/19006586.297

8. Wells JCK, Fewtrell MS. Is body composition important for paediatricians? Archives of Disease in Childhood. 2008; 93: 168-172.

9. Carrasco F, Reyes E, Rilmer O, Ríos F. Exactitud del Índice de Masa Corporal en la predicción de la adiposidad medida por impedanciometría bioeléctrica. Archivos Latinoamericanos de Nutrición. 2004; 54(3), 280-286.

10. Moreira O, Alonso-Aubin DA, Patrocinio de Oliveira CE, Candia-Luján R, De Paz JA. Métodos de evaluación de la composición corporal: una revisión actualizada de descripción, aplicación, ventajas y desventajas. Archivos de Medicina del Deporte. 2015; 32 (6): 387-394.

11. Malina RM, Bouchard C, Bar-Or O. Growth, maturation and physical activity. En Body composition. Champaign: Human kinetics. 2004, pp. 101-119.

12. Garrow JS. New approaches to body composition. Am J Clin Nutr. 1982; 35: 11521158 .

13. Wells JCK, Fewtrell M. Measuring body composition. Archives of Disease in Childhood. 2006; 91(7): 612-617. Disponible en: https://adc.bmj.com/content/91/7/612

14. Kyle UG, Boseaeus I, De Lorenzo AD, Deurenberg P, Elia M, Gómez JM, Heitmann BL, Kent-Smith L, Melchior JC, Pirlich M, Scharfetter H, Schols AM, Prchard C. Bioelectrical impedance analysis-Part I: Review of principles and methods. Clinical Nutrition. 2004 octubre; 23(5), 1226-1243.

15. Kyle UG, Boseaeus I, De Lorenzo AD, Deurenberg P, Elia M, Gómez JM, Heitmann BL, Kent-Smith L, Melchior JC, Pirlich M, Scharfetter H, Schols AM, Prchard C. Bioelectrical impedance analysis - Part II: Utilization in clinical practice. Clinical Nutrition. 2004 diciembre; 23(6), 1430-1453.

16. Lee SY, Gallagher D. Assessment methods in human body composition. Curr Opin Clin Nutr Metab Care. 2008 septiembre; 11: 566-572.

17. Nutrition AJoC. Bioelectrical impedance analysis in body composition measurement: National Institutes of Health Technology Assessment Conference Statement. Am J Clin 
Nutr. 1996 septiembre; 63 (suplemento), 524S-524S. DOI: http://dx.doi.org/10.1093/ ajcn/64.3.524S

18. Mattsson S, Thomas BJ. Development of methods for body composition studies. Phys Med Biol. 2006 julio; 51: R203-28.

19. Sant'Anna M, Priore SE, Franceschini S. Métodos de avaliação da composição corporal em crianças. Revista Paulista de Pediatria. 2011; 27 (3), 315-321.

20. Baumgartner RN, Chumela WC, Roche AF. Bioelectric impedance phase angle and body composition. Am J Clin Nutr. 1988 julio; 48 (1): 16-23.

21. Llames L, Baldomero V, Iglesias ML, Rodota LP. Valores del ángulo de fase por bioimpedancia eléctrica-Estado nutricional y valor pronóstico. Nutrición Hospitalaria. 2013 abril; 28 (2), 286-285. DOI: http://dx.doi.org/10.3305/nh.2013.28.2.6306

22. Madsen C, Mogensen P, Thomas N, Christensen DL, Bygbierg IC, Mohan V, Inbakumari M, Nadig SV, Alex R, Geeranjali FS, Westgate K, Brage S, Vaag A, Grunnet LG. Effects of an outdoor bicycle-based intervention in healthy rural Indian men with normal and low birth weight. Journal of Developmental Origins of Health and Disease. 2014 febrero; 6 (1): 27-37. DOI: http://dx.doi.org/10.1017/S2040174414000609

23. Saladino C. The efficacy of Bioelectrical Impedance Analysis (BIA) in monitoring body composition changes during treatment of restrictive eating disorder patients. Journal of Eating Disorders. 2014; 42 (1): 34.

24. Camina Martín MA, de Mateo Silleras B, Nescolarde Selva L, Barrera Ortega S, Domínhez Rodríguez L, Rendon del Río MP. Bioimpedance vector analysis and conventional bioimpedance to assess body composition in older adults with dementia. Nutrition. 2015 enero; 31(1), 155-159. DOI: http://dx.doi.org/10.1016/j. nut.2014.06.006.

25. Esco MR, Snarr Rl Leatherwood MD, Chamberlain NA, Redding ML, Flatt AA, Moon JR, Williford HN. Comparison of total and segmental body composition using DXA and multifrequency bioimpedance in collegiate female athletes. Journal of Strength and Conditioning Research. 2015 abril; 29 (4), 918-925. DOI: http://dx.doi.org/10.1519/ JSC.0000000000000732.

26. Núnez C, Carvajal A, Turmero E, Moreiras O. Contribución al estudio de la composición corporal de un grupo de mujeres jóvenes mediante análisis de impedancia bioeléctrica. Nutrición Hospitalaria. 1994; 9: 262-267.

27. Ayvaz G. Methods for Body Composition Analysis in Adults. The Open Obesity Journal. 2011; 3(1) 62-69.

28. Kenney, WL, Wilmore JH, Costill DL. Fisiología del deporte y el ejercicio. 5. a ed. Bogotá: Panamericana; 2014.

29. Cometti G. Los métodos modernos de musculación. 3. ${ }^{a}$ ed. Barcelona: Paidotribo; 2001. 